\title{
Degree of Myelination (g-ratio) of the Human Hypoglossal Nerve
}

\author{
D. Campos ${ }^{1,2, *}$ \\ J.N. Páfaro ${ }^{1}$ \\ G.R. Goulart ${ }^{2}$ \\ C.H. Silveira ${ }^{2}$ \\ P.A.M. Cella ${ }^{1}$ \\ T. Malysz 3,4 \\ G.P. $J o t z^{3,5}$
}

${ }^{1}$ Departamento de Ciências Básicas da Saúde, Universidade Federal de Ciências da Saúde de Porto Alegre, Porto Alegre, RS, Brazil

2 Departamento de Biologia e Farmácia, Universidade de Santa Cruz do Sul, Santa Cruz do Sul, RS, Brazil

${ }^{3}$ Departamento de Ciências Morfológicas, Instituto de Ciências Básicas da Saúde, Universidade Federal do Rio Grande do Sul, Porto Alegre, RS, Brazil

${ }^{4}$ Programa de Pós-graduação em Neurociências, Instituto de Ciências Básicas da Saúde, Universidade Federal do Rio Grande do Sul, Porto Alegre, RS, Brazil

5 Programa de Pós-graduação em Ciências da Saúde, Universidade Federal de Ciências da Saúde de Porto Alegre, Porto Alegre,

RS, Brazil

J Morphol Sci 2018;35:25-27.
Address for correspondence D. Campos, Departamento de Ciências Básicas da Saúde, Universidade Federal de Ciências da Saúde de Porto Alegre - UFCSPA, Avenida Sarmento Leite, 245, CEP 90050-170, Porto Alegre, RS, Brazil (e-mail: dcampos@ufcspa.edu.br).

\begin{abstract}
Keywords

- hypoglossal nerve

- g-ratio

- axon diameter

- myelinated fiber diameter

- human

Introduction The degree of myelination ( $g$-ratio) can be useful to the evaluation of the fiber morphology during peripheral nerve regeneration and in studies in the area of microsurgery. Therefore, the aim of this analyze was to investigate the g-ratio of the human hypoglossal nerve.

Materials and Methods The hypoglossal nerve was bilaterally analyzed in human specimens obtained from necropsies (6 subjects). The nerves were analyzed using histology, and the morphometric parameters (axon diameter and myelinated fiber diameter) were measured using Image Pro-Plus Software 6.0.

Results Quantitative comparison of the g-ratio demonstrated no statistically significant difference between the left and right human hypoglossal nerves. In addition, the values of the g-ratio of both hypoglossal nerves were considerably higher than the normal values for the peripheral nerve fibers. This demonstrates a low degree of myelination of the hypoglossal nerve, bilaterally.

Conclusion Knowledge of the variations of the human hypoglossal nerve, are clinically important especially in the area of microsurgery (techniques used for reinnervation).
\end{abstract}

\section{Introduction}

A variety of ways of transferring and suturing (microsurgery/ techniques used for reinnervation) the hypoglossal nerve (XII) to the distal segment of the facial nerve have been reported. ${ }^{1,2}$ However, while the macroscopic anatomy of the XII nerve ${ }^{3}$ and its cervical loop, have been well docu- mented $^{2,4}$ a few studies have been done on the microscopic anatomy of this nerve, especially on the degree of myelination (g-ratio) bilaterally. Recently, some authors ${ }^{5}$ have reported that the g-ratio describes the relationship between axon size and myelin thickness, and deviations in the g-ratio are thought to be involved in abnormal development and received

March 13, 2017

accepted

February 28, 2018
DOI https://doi.org/

10.1055/s-0038-1660484. ISSN 2177-0298.
Copyright $(2018$ by Thieme Revinter

Publicações Ltda, Rio de Janeiro, Brazil
License terms

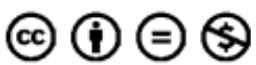


disease. Moreover, the g-ratio can be useful to the evaluation of the relationship between nerve conduction velocity, fiber morphology during peripheral nerve regeneration and in studies in the area of microsurgery. ${ }^{6}$ Therefore, we decide to present here the data about the g-ratio of the human XII nerve (bilaterally) extracted of the histological collection of our research group.

\section{Methodology}

The histological material of nerves were obtained from the collection of the Laboratory of Histology and Pathology of the Universidade de Santa Cruz do Sul, RS, Brazil, these tissues were collected from necropsies (dissected from the point where it crosses the internal carotid artery to its ending at the tongue - bilaterally). Six subjects (males [age $=65.6$ $\pm 11.5]$ ) were used in our study. None of the subjects used in this study had any illness or injury that could alter the fibers of the XII nerve.

\section{Morphometric Measurements}

The g-ratio (degree of myelination) was calculated by measuring the diameter of axons and dividing by the total diameter of that axon plus the surrounding myelin sheath

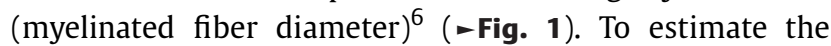
axonal diameter $(\mu \mathrm{m})$, the axonal area of each individual fiber was measured and the value obtained was converted to the diameter of a circle with an equivalent area. The sum of the axonal diameter and myelin sheath represent the myelinated fiber diameter $(\mu \mathrm{m})$. Myelin sheath thickness was estimated using the measurement tools of the Image ProPlus Software [Image Pro-Plus 6.0; Media Cybernetics, Silver Spring, MD, USA] (four vertical and horizontal lines in each fiber counted in areas of interest) (-Fig. 1). All statistical analyses were performed using GraphPad Prism 5.0 software. The comparisons between the right and left XII nerve from each subject were performed using a paired $t$ test.

\section{Results}

The main result found in our analyze is the significant difference in axonal diameter and myelinated fiber diameter between the left and right human XII nerves, the left XII nerve being bigger when compared with the right XII nerve. The axon diameter in the left nerve $(9.2 \pm 0.3 \mu \mathrm{m})$ was found to be $8.7 \%$ larger than that on the right nerve $(8.4 \pm 0.4 \mu \mathrm{m})$ $(p=0.0001)$ (-Fig. 1).

There was also a significant difference between the myelinated fiber diameter of the left $(10.2 \pm 0.7 \mu \mathrm{m})$ and right (9.6 $\pm 0.7 \mu \mathrm{m})$ nerves, the former being found to be $5.9 \%$ larger $(p=0.0001)$. On the other hand, quantitative comparison of the g-ratio demonstrated no statistically significant difference between the left $(0.90 \pm 0.1)$ and right $(0.88 \pm 0.1)$ nerves $(p=0.0798)$ (-Fig. 1$)$.

\section{Discussion}

For this study, were used histological slides of the XII nerve, made previously by our research group for the comparison of numerous morphometric parameters between the recurrent laryngeal nerve and the XII nerve of humans. However, in this study, ${ }^{7}$ no comparison between the g-ratio of right and left XII nerve was performed. Thus, to search for more scientific articles that could be confronted with our results, we perform a basic search in the following scientific databases:

- PubMed (NCBI, 2017), ${ }^{8}$

- Bireme (BVS, 2017), ${ }^{9}$

- HighWire (2017). ${ }^{10}$

The search terms were as follows: human hypoglossal nerve, degree of myelination and g-ratio. Our research shows that there are no human studies in the literature showing comparative data between the g-ratio of the left and right XII nerves.
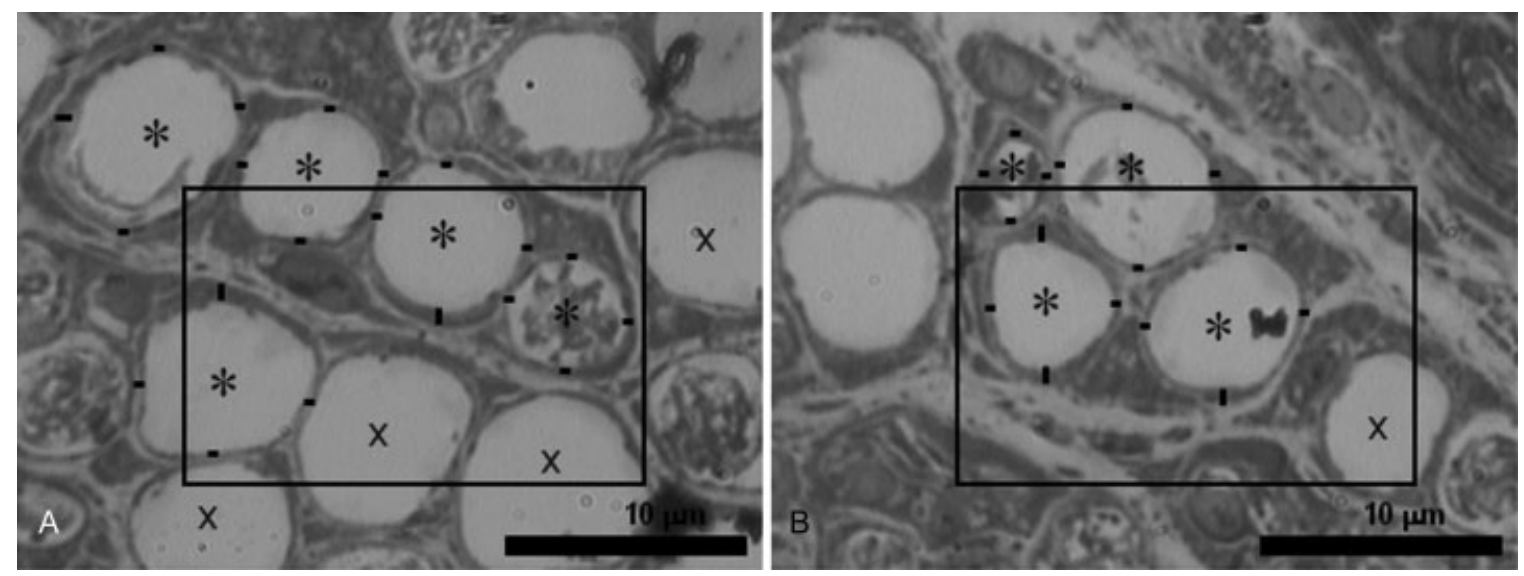

Fig. 1 Images demonstrating the differences between the left (A) and right (B) nerves in terms of myelinated fiber diameter. Representation of one of the fields (areas of interest) used in the study. The fibers located inside this square or intersected by the upper and/or left edge of the areas of interest were counted $\left({ }^{*}\right)$; the fibers intersected by the lower and/or right edge of the areas of interest were not counted (x). Images demonstrating the myelin sheath thickness of fibers were counted within the areas of interest, calculated by means of a specific software tool (four vertical and horizontal lines in each fiber) of the Image Pro-Plus 6.0; Media Cybernetics, Silver Spring, MD, USA. Semithin sections (1 $\mu$ m) were stained with toluidine blue. 
However, although we have found no studies in the literature that showed some data that could be compared with our results, these limitations do not change the main goals and results of our study, to provide new information and basic knowledge about the human XII nerve bilaterally, which may be essential for understanding and improving the techniques used for reinnervation.

In addition, in our study, the values of the g-ratio of both hypoglossal nerves were considerably higher than the normal values (between 0.6 and 0.7 ) for the peripheral nerve fibers. ${ }^{11}$ This demonstrates a low degree of myelination of the hypoglossal nerve, bilaterally.

\section{Acknowledgments}

This study was supported by Conselho Nacional de Desenvolvimento Científico e Tecnológico (CNPq) and Coordenação de Aperfeiçoamento de Pessoal de Nível Superior (CAPES).

\section{References}

1 Slattery WH III, Cassis AM, Wilkinson EP, Santos F, Berliner K. Sideto-end hypoglossal to facial anastomosis with transposition of the intratemporal facial nerve. Otol Neurotol 2014;35(03):509-513 Doi: 10.1097/MAO.0b013e3182936bcf

2 Vacher C, Dauge MC. Morphometric study of the cervical course of the hypoglossal nerve and its application to hypoglossal facial anastomosis. Surg Radiol Anat 2004;26(02):86-90 Doi: 10.1007/ s00276-003-0197-8

3 Çandir N, Develi S, Kilir C, Satar B, Yazar F. Distribution of the hypoglossal nerve at the base of the tongue and its clinical importance in radiofrequency ablation therapy. Turk J Med Sci 2014;44(03):427-431 Doi: 10.3906/sag-1301-63

4 Li M, Liu F, Shi S, Chen S, Chen D, Zheng H. Bridging gaps between the recurrent laryngeal nerve and ansa cervicalis using autologous nerve grafts. J Voice 2013;27(03):381-387 Doi: 10.1016/j. jvoice.2013.01.009

5 West KL, Kelm ND, Carson RP, Does MD. A revised model for estimating g-ratio from MRI. Neuroimage 2016;125:1155-1158 Doi: 10.1016/j.neuroimage.2015.08.017

6 Ikeda M, Oka Y. The relationship between nerve conduction velocity and fiber morphology during peripheral nerve regeneration. Brain Behav 2012;2(04):382-390 Doi: 10.1002/brb3.61

7 de Campos D, do Nascimento PS, Ellwanger JH, et al. Histological organization is similar in human vocal muscle and tongue-a study of muscles and nerves. J Voice 2012;26(06):811.e19-811. e26 Doi: 10.1016/j.jvoice.2011.12.006

8 National Center For Biotechnology Information - NCBI [viewed 13 March 2017]. PubMed 2017. Available from: http://www.ncbi. nlm.nih.gov/pubmed/

9 Biblioteca Virtual Em Saúde - BVS [viewed 13 March 2017]. Bireme. 2017. Available from: http://brasil.bvs.br/

10 Highwire [viewed 13 March 2017]. 2017. Available from: http:// highwire.stanford.edu/lists/freeart.dtl

11 Chomiak T, Hu B. What is the optimal value of the g-ratio for myelinated fibers in the rat CNS? A theoretical approach. PLoS One 2009;4(11):e7754 Doi: 10.1371/journal.pone.0007754 